\title{
Grafting of collagen onto interpenetrating polymer networks of poly(2-hydroxyethyl methacrylate) and poly(dimethyl siloxane) polymer films for biomedical applications
}

\author{
S. M. Rezaei, Z. A. Mohd Ishak* \\ School of Materials \& Mineral Resources Engineering, Engineering campus, University Sains Malaysia, 14300 Nibong \\ Tebal, Penang, Malaysia
}

Received 29 June 2013; accepted in revised form 1 September 2013

\begin{abstract}
Poly(dimethyl siloxane) (PDMS) films were modified using poly(2-hydroxyethyl methacrylate) (PHEMA) by sequential method of interpenetrating polymer networks (IPNs). Collagen (type I) was also linked onto the modified films via methyl sulfonyl chloride. Collagen reacted with the activated groups to obtain covalently linked protein layers. The surface properties of samples were characterised by attenuated total reflectance Fourier transform infrared spectroscopy (ATRFTIR), X-ray photoelectron spectroscopy (XPS), scanning electron microscopy (SEM) and water contact angle measurements. Dynamic Mechanical Thermoanalysis (DMTA) and tensile testing were used to study the physical and mechanical properties of the IPNs.

Grafting of collagen on the surface was confirmed using ATR-FTIR and XPS. The results also showed that the hydrophilicity of modified samples increased as the content of PHEMA increased. The biocompatibility of the PDMS surface was evaluated by culture of fibroblast cells (L929). The collagen-linked surfaces showed significant cell adhesion and growth in comparison with unmodified PDMS samples.
\end{abstract}

Keywords: biocompatible polymers, poly(dimethyl siloxane), interpenetrating polymer network, collagen, hydrophilicity

\section{Introduction}

Polymeric biomaterials have played an important role in medical therapy, finding applications in areas such as implantable medical devices and artificial organs, ophthalmology, dentistry, bone repair, drug delivery systems and many other medical fields. These biomaterials are easily fabricated into products with various shapes at reasonable cost. Despite possessing desirable mechanical and physical properties, these materials have one major drawback, i.e., biocompatibility, that limits their applicability [1]. Depending on the proposed medical application, all biomaterials are evaluated in terms of biocompatibility. Biocompatibility can be defined as the acceptance of an artificial material by the surrounding tissues and the body as a whole. It is commonly accepted that this term means not only a lack of cytotoxic effects, but also positive effects in the sense of biofunctionality $[2,3]$.

PDMS-based materials have been mostly used in biomedical applications as soft tissue substitutes because of their excellent softness, stability and inertness. However, serious problems have occurred when silicone devices are implanted for long periods of time $[4,5]$. These include damage to the tissues in direct contact through mechanical friction

\footnotetext{
${ }^{*}$ Corresponding author, e-mail: zarifin@eng.usm.my

(C) BME-PT
} 
and dense fibrous tissue formation around the surface, without bulk deterioration. On the other hand, cell attachment on PDMS is rather low due to its hydrophobicity [6]. One approach to solving these problems is the fixation of a biocompatible layer on the surface of the polymer to improve cellular interactions by covalent grafting of a coupling agent and suitable macromolecular chains. In recent years, a large number of research groups have studied protein immobilisation on polymer surfaces to improve biocompatibility [7-9]. Fixing of some special biologically active molecules on synthetic materials is of critical importance since they can, in principle, elicit some specific, predictable and controlled responses from the cells seeded on the materials. Among the different types of natural materials, collagen is one of the most important biological macromolecules of the extracellular matrix in tissues. Collagen has been successfully used to produce biomaterials for a wide range of applications, including burn healing and the strengthening of soft tissue [10-14]. In order to covalently link protein molecules on an inert polymer surface such as PDMS, it is necessary to introduce reactive groups such as hydroxyl, carboxyl or amino groups on the polymer surface [15].

Hydrogels with desirable functional groups and high hydrophilicity have received considerable attention as excellent biomaterials. Among the many important hydrogels, PHEMA is the first one that has been successfully employed for biological use. PHEMA is known as a hydrogel with good biomedical properties. Applications of PHEMA are versatile and it has been engaged in injectable polymeric systems, nasal cartilage, ophthalmic applications, drug delivery systems, artificial corneas and wound dressings [16-20]. There are many reports in the literature for improving the surface properties of silicone using hydrogels by laser, plasma, corona discharge and particulate composites methods [21-27]. Another option for modifying PDMS by hydrogels is to form an interpenetrating polymer network [28$31]$. Interpenetrating polymerisation is the only way of combining cross-linked polymers. This technique can be used to combine two or more polymers into a mixture in which phase separation is not as extensive as it would be otherwise [32]. In the present work, PDMS/PHEMA film was prepared via IPN to improve the hydrophilicity of PDMS. The functional groups of PHEMA on the silicone surface were covalently coupled with collagen via a coupling agent to improve the cell adhesion properties of PDMS. Thus, incorporating PHEMA and collagen onto the silicone surface may give silicone substrates new and interesting properties for applications in silicone-based implantable biomaterials.

\section{Experimental}

\subsection{Materials}

The materials used are listed in Table 1. HEMA was redistilled under vacuum. Azobisisbutyronitrile (AIBN) was recrystallised twice from methanol. Ethylen glycol dimethacrtylate (EGDMA) was used as received. Medical grade PDMS was used without any further purification. All other chemicals used in this study were of reagent grade and were used as received.

Table 1. Materials used

\begin{tabular}{|l|l|l|}
\hline Designation & \multicolumn{1}{|c|}{ Description } & \multicolumn{1}{c|}{ Source } \\
\hline HEMA & 2-Hydroxyethyl methacrylate & Merck Co. \\
\hline AIBN & $\alpha, \alpha$-Azoisobutyronitrile & Fluka Co. \\
\hline EGDMA & Ethylene glycol dimethacrylate & Merck Co. \\
\hline- & Toluene & Merck Co \\
\hline- & Ethanol & Riedel Co. \\
\hline PDMS & Silastic MDX4-4210 & Dow Corning Co. \\
\hline Col & Collagen type I & Sigma Aldrich \\
\hline- & Methyl sulfonyl chloride & Sigma Aldrich \\
\hline- & Diethyl ether & Sigma Aldrich \\
\hline
\end{tabular}

\subsection{PDMS vulcanisation}

Medical grade PDMS (Silastic MDX4-4210) is a two-component product. The elastomer component of PDMS was mixed with $10 \% \mathrm{w} / \mathrm{w}$ curing agent part. After thorough mechanical stirring, the mixture was degassed. The PDMS sheets were prepared by compression moulding (GOTECH, GT-7014-A) ( $250 \mathrm{psi} / 1,75 \mathrm{MPa} /, 75^{\circ} \mathrm{C}, 30 \mathrm{~min}$ ), followed by a post-curing process at $120^{\circ} \mathrm{C}$ for a period of $2 \mathrm{~h}$ to establish the physical properties.

\subsection{Preparation of IPN}

The PDMS films were immersed at room temperature for $24 \mathrm{~h}$ in a swelling solution of HEMA (monomer), EGDMA (crosslinker) and AIBN (initiator) in toluene. The swollen samples were suspended in a sealed glass reactor. The initiator and crosslinker concentration were fixed at 0.2 and $2 \mathrm{wt} \%$ with respect to the amount of monomer, respectively. The temperature was then raised and kept at $80^{\circ} \mathrm{C}$ for $3 \mathrm{~h}$ to allow HEMA, EGDMA and 
AIBN to react. The obtained IPNs were kept at $90^{\circ} \mathrm{C}$ for $4 \mathrm{~h}$ to complete polymerisation of the monomer. The produced IPNs were immersed in ethanol for $24 \mathrm{~h}$, followed by Soxhlet extraction in distilled water for $48 \mathrm{~h}$ to remove homopolymers and unreacted monomers. The specimens were then dried until constant weight under vacuum at $50^{\circ} \mathrm{C}$. The PHEMA content of the IPNs was calculated as shown by Equation (1):

$$
\text { PHEMA content }[\%]=\frac{w_{\text {IPN }}-w_{0}}{w_{\text {IPN }}} \cdot 100
$$

where $w_{\text {IPN }}$ is the weight of the IPN and $w_{0}$ is the weight of the unmodified PDMS sample. Samples of IPNs, which contained approximately $30 \%$ PHEMA, were then selected for collagen grafting. These compositions have been reported as the optimum composition to give good biocompatibility and physical and mechanical properties [6].

\subsection{Collagen grafting}

IPN films were introduced into a glass tube containing $2 \mathrm{~mL}$ of methyl sulfonyl chloride and $20 \mathrm{~mL}$ of diethyl ether to activate hydroxyl groups in the PHEMA chains. After incubation at $20^{\circ} \mathrm{C}$ for $3 \mathrm{~h}$, the activated films were immersed into a collagen solution at $4^{\circ} \mathrm{C}$ for $24 \mathrm{~h}$ to allow the activated hydroxyl groups in the PHEMA chains and the amino groups in the protein to form covalent bonds. The concentration and $\mathrm{pH}$ of the collagen solution were $1 \mathrm{mg} \cdot \mathrm{mL}^{-1}$ and 3.0, respectively. Collagen-grafted PDMS films were rinsed with distilled water and the samples were stored at $4^{\circ} \mathrm{C}$ before use. Figure 1 shows the schematic diagram of the reaction of collagen-grafted PDMS/PHEMA IPN films.

\subsection{Characterisation}

Stress-strain measurements were carried out using an Instron (3366) tensile tester on dumbbell-shaped samples with a thickness of $1 \mathrm{~mm}$. The tests were performed with a speed of $50 \mathrm{~mm} \cdot \mathrm{min}^{-1}$ at room tem- perature. The hardness of the samples was measured using a Shore-A durometer. Five specimens were measured for each composition. The elongation at break, tensile strength and hardness were obtained by averaging the results of five specimens. To characterise the surface of modified samples, infrared spectra were obtained on a Fourier transform infrared (FT-IR) spectrophotometer (Perkin Elmer-Spectrum one) using an attenuated total reflection (ATR) method. The collagen grafting on the PDMS/ PHEMA IPN surface was analysed by X-ray photoelectron spectroscopy (XPS). An Omicron X-PS system (ELS5000, Germany) with an unmonochromated aluminum $\mathrm{Al} \mathrm{K}_{\alpha}$ source $(1486: 6 \mathrm{eV})$ was run to record the XPS spectra of the samples. The emission angle from the X-ray detector with respect to the sample surface was $90^{\circ}$. Scanning electron microscopy (FESEM, ZEISS-SUPRA 35 VP) was performed on gold-coated samples using a sputter coater. A SEM typically operating at $10 \mathrm{kV}$ was employed for morphological observation. Static water contact angles were investigated on the swollen samples in water for $24 \mathrm{~h}$ using the sessile drop method with contact-angle measurement equipment (KRUSS- K12). The swollen samples were dried with filter paper before water contact angle measurements were acquired. All results of the water contact angles were the average value of five measurements on different parts of the sample.

Dynamic mechanical thermal analysis measurements (DMTA) were conducted using a PolymerLab DMTA analyser (Mettler Toledo DMA 861) at $10 \mathrm{~Hz}$ over a temperature range of -150 to $150^{\circ} \mathrm{C}$ and at a heating rate of $2^{\circ} \mathrm{C} / \mathrm{min}$ in the bending mode under a dry nitrogen flow.

\subsection{Cell culture study}

Cell adhesion was determined with the mouse L929 fibroblast cell line (purchased from ATTC). The cells were maintained in Dulbecco's modified eagle's medium (DMEM) supplemented with $100 \mathrm{IU} / \mathrm{mL}$

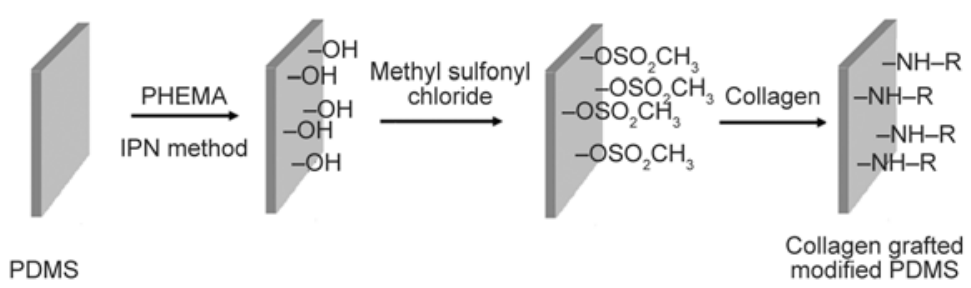

Figure 1. Schematic diagram of the reaction of the collagen grafted PDMS/PHEMA IPN film surface. HN-R represents a collagen molecule 
penicillin, $100 \mu \mathrm{g} / \mathrm{mL}$ streptomycin (Gibco BRL) and $10 \%$ fetal calf serum (Gibco BRL). A routine subculture was used to maintain the cell line. The cells were incubated in a humidified atmosphere of $5 \% \mathrm{CO}_{2}$ at $37^{\circ} \mathrm{C}$. After 1 week of incubation, the monolayer was harvested by trypsinisation. A cell suspension of $3 \cdot 10^{5}$ cells $/ \mathrm{mL}$ was prepared before seeding. The samples were sterilised in $70 \%$ alcohol and washed in culture medium before the cell seeding procedure. They were then placed in a multi-well tissue culture polystyrene plate with the cell suspension and maintained in an incubator for $72 \mathrm{~h}$. After incubation, the samples were removed from the incubator and washed immediately in phosphate-buffered saline (PBS). The cells were fixed in graded alcohol (60, 70, 80 and 99\%) and stained with $5 \%$ Giemsa for optical microscopic examinations (OLYMPUS- BX51M). All samples were run in duplicate and approximately 10 objective fields were randomly chosen in the central and peripheral regions of each sample.

\section{Results and discussion}

\subsection{Tensile properties}

Tensile tests were carried out to study the hydrogel effect on the mechanical properties of the PDMS, and the results are shown in Table 2. As can be seen, the IPN samples up to approximately $30 \mathrm{wt} \%$ of PHEMA reveal good tensile strengths and elongations at break in comparison to the PDMS. The tensile strength and elongation at break of the PDMS/ PHEMA IPN samples were gradual lower than that of PDMS, based on the weak mechanical properties of PHEMA, these results are acceptable because the hydrophilicity of these IPNs were significantly improved. In addition, for samples with approximately $31.7 \mathrm{wt} \%$ PHEMA, tensile strength and elongation at break were about $15 \%$ lower than that of the unmodified sample. The hardness values of the IPN systems were in agreement with the results of

Table 2. Hardness (Shore A), tensile strength and elongation at break percentage of PDMS/PHEMA IPN

\begin{tabular}{|l|c|c|c|}
\hline $\begin{array}{c}\text { PHEMA \% } \\
\text { in IPN }\end{array}$ & Hardness & $\begin{array}{c}\text { Tensile strength } \\
\text { [MPa] }\end{array}$ & $\begin{array}{c}\text { Elongation } \\
{[\text { [\%] }}\end{array}$ \\
\hline 0 & $35 \pm 0.16$ & $5.4 \pm 0.03$ & $493 \pm 1.41$ \\
\hline 10.5 & $37 \pm 0.35$ & $5.1 \pm 0.06$ & $475 \pm 2.47$ \\
\hline 19.4 & $40 \pm 0.57$ & $4.8 \pm 0.12$ & $443 \pm 3.35$ \\
\hline 31.7 & $43 \pm 0.16$ & $4.5 \pm 0.02$ & $427 \pm 1.27$ \\
\hline 40.8 & $51 \pm 0.48$ & $3.6 \pm 0.08$ & $340 \pm 4.26$ \\
\hline
\end{tabular}

the elongation measurements because harder polymers show lower elongations at break.

\subsection{Surface characterisation by ATR-FTIR}

Surface characterisation of the PDMS samples was performed by comparing the ATR-FTIR spectra of unmodified and modified PDMS. Figure 2 shows the spectra of the samples. The absorption bands at approximately $1005 \mathrm{~cm}^{-1}$ are the characteristic absorption bands for - $\mathrm{Si}-\mathrm{O}-\mathrm{Si}-$ groups of PDMS polymers (Figure 2 curve a). The absorption bands appearing at approximately $1715 \mathrm{~cm}^{-1}$ are the characteristic absorption bands for carbonyl groups, which confirm the presence of PHEMA on the surface (Figure 2 curve b). A broad adsorption that appeared at $3100-3600 \mathrm{~cm}^{-1}$ confirms that the collagen was linked onto the functional group of the PDMS/ PHEMA IPN by the coupling agent (Figure 2 curve c). This figure indicated that $\sim 3350 \mathrm{~cm}^{-1}$ was the adsorption peak of hydrogen bonding and $\sim 3150 \mathrm{~cm}^{-1}$ was the peak for $\mathrm{N}-\mathrm{H}$ bonds of collagen. In addition, the absorption at $\sim 1600 \mathrm{~cm}^{-1}$ confirms the presence of amide bonds on the collagengrafted film surfaces that were associated with carbonyl groups. Hence, it was concluded that grafting occurred on the film surface.

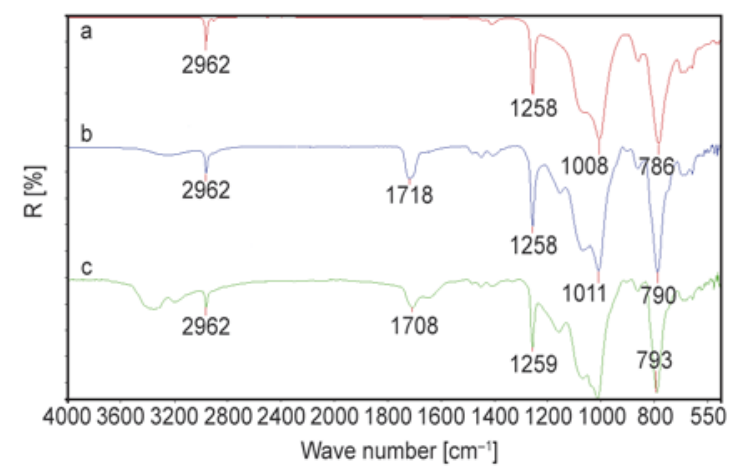

Figure 2. FTIR-ATR spectra of (a) unmodified PDMS; (b) PDMS/PHEMA IPN with $31.7 \mathrm{wt} \%$ PHEMA; and (c) collagen grafted PDMS/PHEMA IPN

\subsection{XPS study}

Figure 3a depicts the XPS wide scan spectrum of the PDMS surface. It shows a spectrum consisting of the electrons of oxygen (O 1s), carbon (C 1s), and silicones (Si 2s and Si 2p). The peaks of these elements occurred in the regions around 533, 285, 153 and $103 \mathrm{eV}$, respectively. The spectrum of the PDMS/PHEMA IPN film surface (Figure 3b) also 

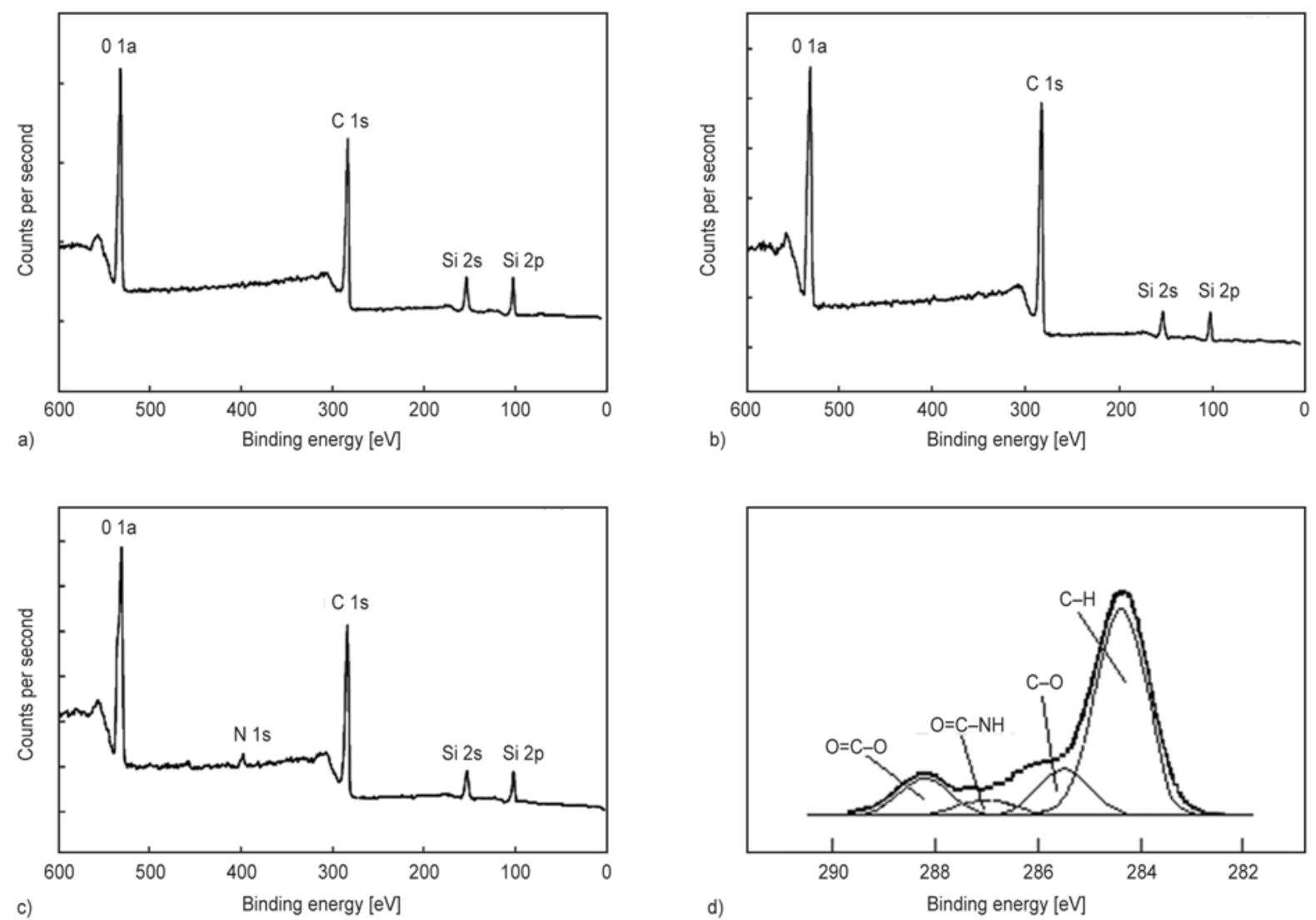

Figure 3. XPS wide scan spectra of (a) unmodified PDMS; (b) PDMS/PHEMA IPN with 31.7 wt\% PHEMA; (c) collagengrafted PDMS/PHEMA IPN and (d) high resolution C1s spectrum for the collagen grafted PDMS/PHEMA IPN

showed the same peaks as the PDMS surface. However, the relative intensity of the carbon and oxygen peaks varied after formation of the PHEMA IPNs. In contrast, a new peak corresponding to $\mathrm{N} 1 \mathrm{~s}$ at around $400 \mathrm{eV}$ appeared in the spectrum of the collagen-grafted film surface (Figure $3 \mathrm{c}$ ). The wide scan and low-resolution spectra were performed to establish which elements were available on the various surfaces. $\mathrm{N}$ ls was only present on the modified surface grafted with collagen. Therefore, collagen was successfully introduced onto the modified surfaces based on this spectrum. Figure $3 \mathrm{~d}$ shows the respective $\mathrm{C} 1 \mathrm{~s}$ core-level spectra of the samples. In the case of the PDMS/PHEMA IPN film surface, the $\mathrm{C} 1 \mathrm{~s}$ core level spectrum contained three major peak components with binding energies at 284.6, 286.2 and $288.6 \mathrm{eV}$, corresponding to carbon atoms of hydrocarbons, carbon atoms with a single bond to oxygen and carbon atoms in carbonyl groups. After collagen grafting onto the film surface, the $\mathrm{C} 1 \mathrm{~s}$ core level was curve-fitted with four peak components with binding energies at $284.6 \mathrm{eV}$ for the $\mathrm{C}-\mathrm{H}$ species, $286.2 \mathrm{eV}$ for the $\mathrm{C}-\mathrm{O}$ species, $287.4 \mathrm{eV}$ for the $\mathrm{O}=\mathrm{C}-\mathrm{NH}-$ species and $288.6 \mathrm{eV}$ for the $\mathrm{O}-\mathrm{C}=\mathrm{O}$ species.

\subsection{Contact angle study}

One of the best ways to improve the hydrophilicity of a given polymer surface is to allow water to engage its preferred interaction with the surface, i.e., the short-range hydration, acid-base and hydrogen bonding interactions. The presence of hydrophilic groups on the surface of the PDMS/PHEMA IPNs was confirmed by surface characterisation methods. The wettability of the unmodified and modified films was studied using static water contact angles. As can be seen in Figure 4, the surface wettability of the modified PDMS film was obviously increased compared with the unmodified PDMS film, which showed a relatively high contact angle. The water contact angle of the pure PDMS surface was $104.6^{\circ}$; while after modification, the water contact angle of PDMS/PHEMA IPN with $31.7 \mathrm{wt} \%$ PHEMA dropped to $58.6^{\circ}$. Increasing PHEMA content in the IPNs was observed to decrease the contact angles of the samples. The water contact angles for various 


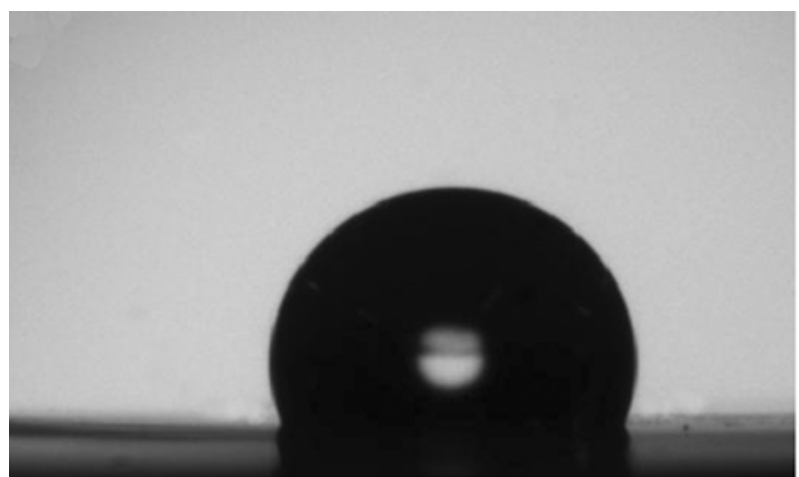

a) $31.7 \mathrm{wt} \%$ PHEMA

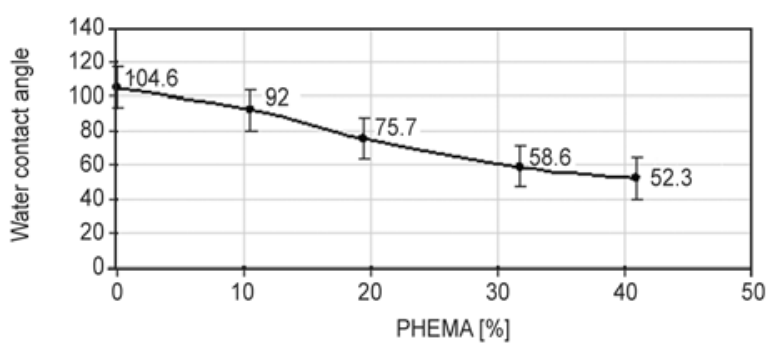

Figure 5. Water contact angle of the unmodified and modified PDMS IPNs films with different amounts of PHEMA

amounts of PHEMA IPN films were measured to examine the change in wettability of the samples. In Figure 5, it can be seen that the IPN films showed a considerable decrease in water contact angle compared to the unmodified PDMS. The water contact angle dramatically decreased from 104.6 to $52.3^{\circ}$ as the content of PHEMA increased. Furthermore, the contact angle for collagen linked onto IPN films was $65^{\circ}$. In fact, the contact angle of the collagenlinked IPN film slightly increased. Consequently, the hydrophilicity of the modified PDMS film was maintained. The wettability of the PDMS film was therefore increased in each of the modified samples. The basis of surface hydrophilisation is to maximise hydration and hydrogen bonding interactions. Hydroxyl, carbonyl and carboxyl groups contain lone pairs, unshared electrons and asymmetric charge distributions. Many types of oxygen-containing organic functional groups can interact with water more effectively than the methyl groups of PDMS. For these reasons, higher PHEMA-containing IPNs have lower water contact angles.

\subsection{Morphological study}

The surface appearance of the unmodified and PDMS/PHEMA IPN samples are shown in scan-

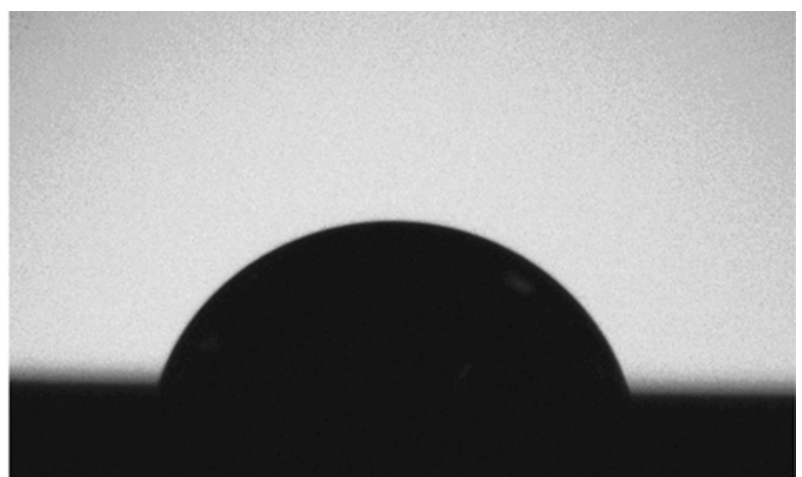

b)

(a) unmodified PDMS and (b) PDMS/PHEMA IPN with ning electron micrographs in Figure 6a and 6b. Both unmodified and modified samples had a smooth surface without any contrast. As can be seen, there was no difference between the unmodified and modified PDMS morphology in accessible magnification of the SEM by this method. This indicates that the dimensions of the hydrophilic phase were smaller than the micron scale. According to the nature of sequential IPNs, the distribution of monomer before establishment as a second network is the molecular dimension [32]. The second network in the composition of any percentage that functions as a dispersed phase is submicron and will not be detectable by SEM in surface appearance of samples. However, as shown in Figure 6d, the SEM micrograph of the cross section IPN sample clearly confirms the appearance of two phases. It can be seen that PHEMA as the second network are homogeneously distributed throughout the PDMS film. The presence of distributed PHEMA in the PDMS matrix increases the hydrophilicity of the IPN samples that is in agreement with the water contact angle results. Additionally, Figure 6c shows the cross section of pure PDMS sample that there is no additional phase. The surface morphology of collagen grafted PDMS/ PHEMA IPN was observed by SEM (Figure 6e). A comparative morphology was made between collagen grafted IPN film and PDMS films that the surface morphology of collagen grafted IPN were different.

\subsection{Dynamic mechanical thermal analysis (DMTA)}

Figure 7 shows the DMTA results of the PDMS and PDMS/PHEMA IPN. As can be seen, in the area of the glass transition of the components, the curve corresponding to the PDMS exhibits a distinct $\tan \delta$ 


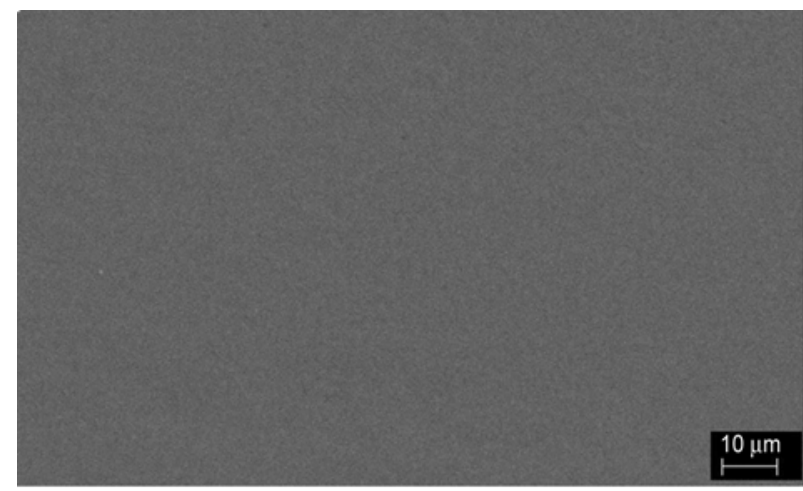

a)

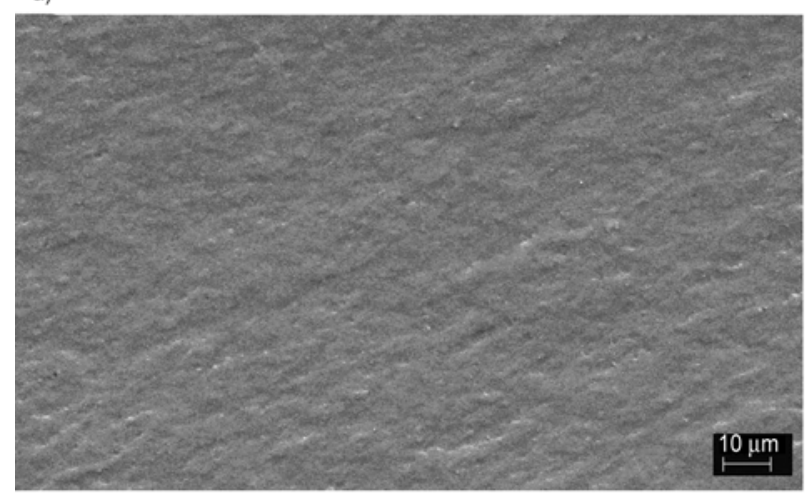

c)

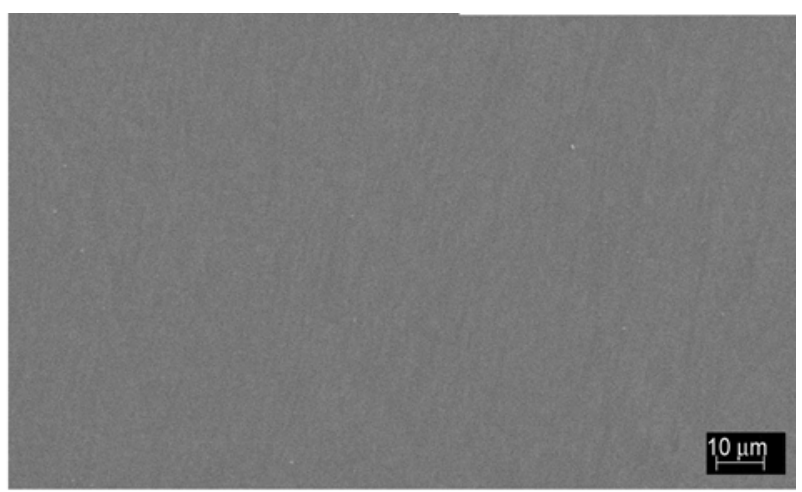

b)

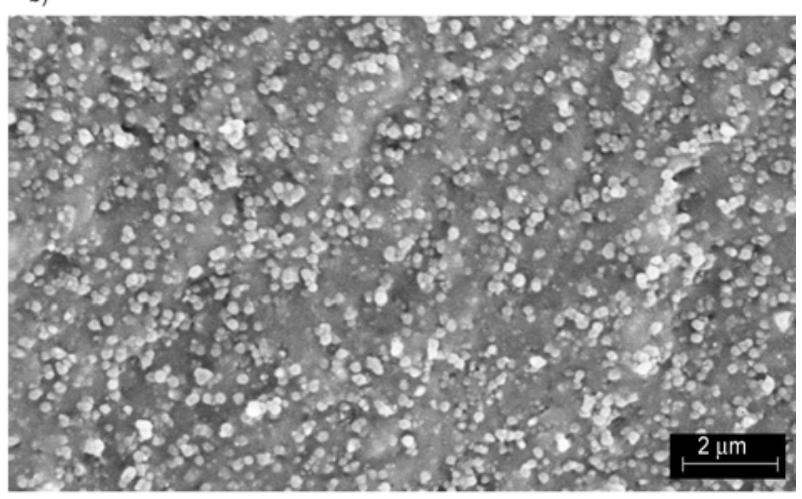

d)

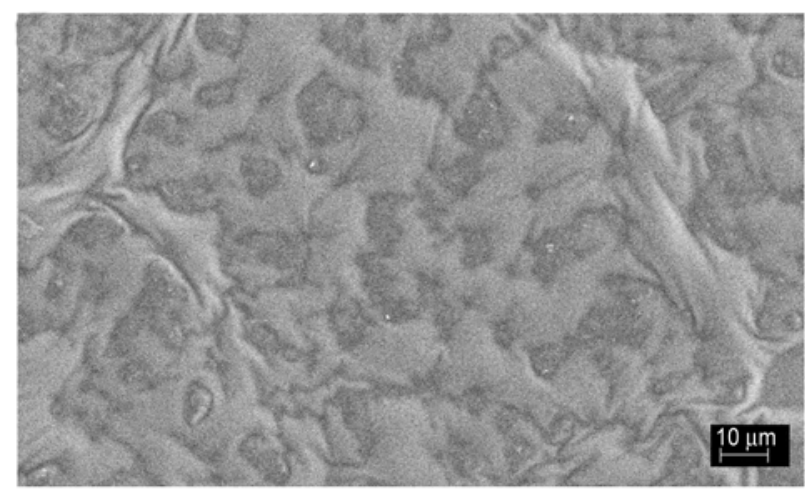

e)

Figure 6. SEM showing surface appearance of (a) unmodified PDMS; (b) PDMS/PHEMA IPN with 31.7 wt \% PHEMA; (c) cross section of unmodified PDMS; (d) cross section of PDMS/PHEMA IPN with 31.7 wt\% PHEMA; and (e) collagen grafted PDMS/PHEMA IPN

maximum at $-37^{\circ} \mathrm{C}$ (Figure $\left.7 \mathrm{a}\right)$, whereas for the PHEMA IPN, the glass-rubber transition occurs at $136^{\circ} \mathrm{C}$ (Figure $7 \mathrm{c}$ ). The DMTA curves corresponding to IPNs with large PHEMA contents depict an obvious maximum in the region of the PHEMA $\alpha$ transition (Figure 7d), however for small PHEMA contents, the curves depict a low maximum (Figure $7 \mathrm{~b}$ ). The storage modulus curves clearly show that there are two $T_{\mathrm{g}} \mathrm{i}$ in the IPNs samples and these compounds are two phase polymeric system. The shapes of the temperature dependence of $\tan \delta$ are typical for two-phase polymeric systems with incompatible components. Moreover, the lack of shifting maximum $\tan \delta$ for PHEMA in IPNs from their position indicated substantially complete phase separation in IPNs form. The height of the damping peaks for each phase varied with IPN composition, while there was no approaching temperature in maximum $\tan \delta$ between two components. As can be seen, DMTA results of IPNs display two main relaxations corresponding to the relaxations of PDMS and PHEMA, indicating that the separation phase occurs during these syntheses. The strong decay of $E^{\prime}$ from $-37^{\circ} \mathrm{C}$ followed by a second decay of $136^{\circ} \mathrm{C}$ allows to assume that the continuous phase is PDMS and PHEMA macro domains dispersed in the con- 

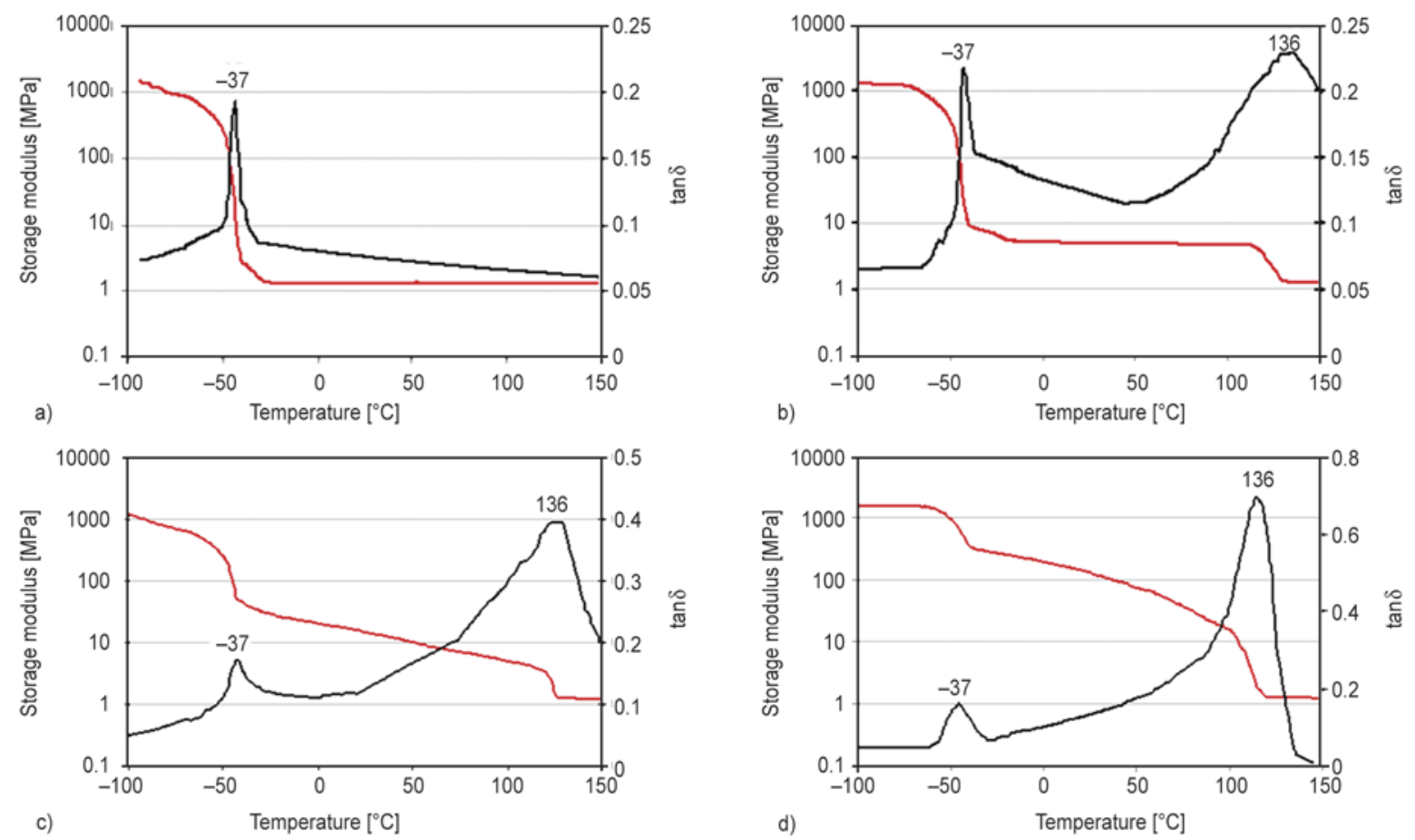

Figure 7. DMTA results curve of PDMS and PDMS/PHEMA IPN (a) PDMS, (b) PDMS/PHEMA IPN with 10.5 wt\% PHEMA, (c) $31.7 \mathrm{wt} \%$ PHEMA, and (d) $40.8 \mathrm{wt} \%$ PHEMA

tinuous phase. This result is in agreement with the general assumption that the continuous phase in IPN is usually the cross-linked phase which is formed first and that second polymer is the dispersed phase [33].

\subsection{Cell attachment and growth study}

The cellular behaviour of a biomaterial is an important factor in determining its biocompatibility. The whole process of adhesion of the cells after contact with biomaterials consists of cell attachment, spreading and growth.

As mentioned earlier, before testing the biological response of the materials, IPN films were immersed in ethanol followed by Soxhlet extraction in distilled water in order to eliminate residual monomers that are potentially toxic.

The optical micrographs of the in vitro cell culture samples are presented in Figure 8. It can be seen that L929 fibroblast cells was attached to the PHEMA IPN surface (Figure 8b) and had grown without eliciting a toxic response. The cell areas attached on the film surface increased with the presence of PHEMA in the PDMS network. This indicated that the attached cells on the PHEMA IPN supported a higher degree of cell spreading and become flat in comparison with the unmodified PDMS (Fig- ure 8a). The fibroblast cells appeared to be attached on the surface of the PHEMA IPN, whereas during the same culture period, the fibroblast cells on the unmodified PDMS were almost round. The difference between cell appearances was likely to be a function of surface chemistry and surface morphology. From a thermodynamic point of view, only materials with a minimal surface tension can provide good adhesive opportunities for cells. As seen in Figure 5, the water contact angle of the PDMS surface decreased with increasing incorporation of PHEMA into the PDMS network. The degree of cell spreading on the PHEMA IPN increased compared with unmodified PDMS. The higher degree of cell spreading on the PHEMA IPN may be attributable to the change in surface wettability of the PDMS surface. As can be seen in Figure 8c, cell adhesion, spreading and growth onto the collagen-grafted onto IPN films was better than with the IPN films. This was attributed to the presence of collagen, which provides a suitable substrate for cell growth. The proliferation of cells onto films grafted with collagen was normal. From the above results, the collagen-grafted films were shown to enhance the attachment and growth capability of the cells. Although wettability is an important factor for cell 

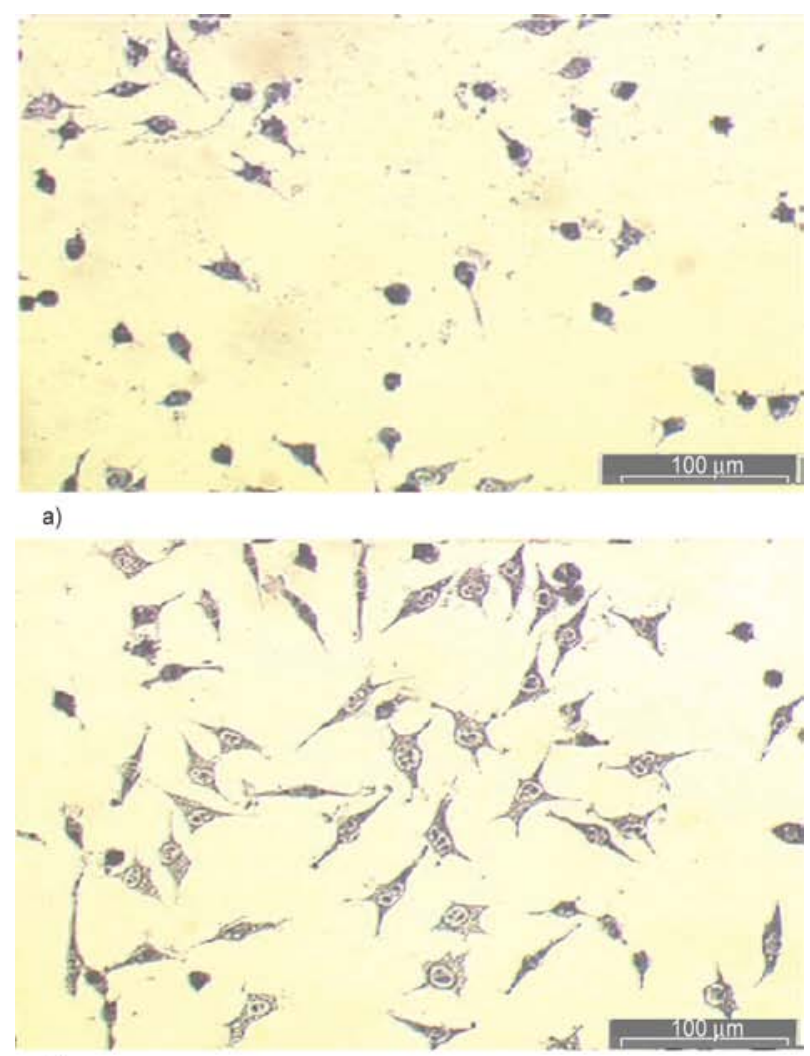

b)

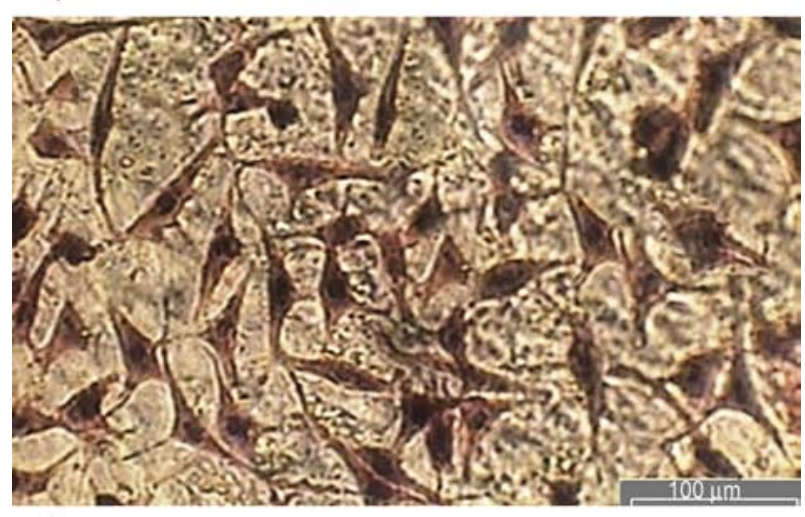

c)

Figure 8. Optical micrographs of cell culture on (a) unmodified PDMS; (b) PDMS/PHEMA IPN with $31.7 \mathrm{wt} \%$ PHEMA; and (c) collagen grafted PDMS/PHEMA IPN

adhesion, spreading and growth onto polymer surfaces, surface chemistry or charge character are also very important factors.

\section{Conclusions}

Collagen-grafted PDMS/PHEMA IPN film was prepared to improve the biocompatibility of PDMS for biomedical applications. PHEMA hydrogel was first introduced onto the PDMS film by the IPN method. Through sulfonyl chloride chemistry, the reactive groups were subsequently used to graft collagen type I. XPS spectra and ATR-FTIR measurements confirmed the collagen grafting on the film surface. SEM and water contact angle surface analysis were performed to confirm the modification process. DMTA results of IPNs displayed two main relaxations corresponding to the relaxations of PDMS and PHEMA, indicating that the separation phase occurs in PDMS/PHEMA IPN system. The wettability of modified PDMS surfaces with and without collagen grafting was enhanced. However, the water contact angle of the PHEMA IPN films was less than that the collagen-grafted IPN film because collagen is a tendency hydrophobic material. Additionally, collagen-grafted film surfaces showed significant cell adhesion and growth in comparison with the PDMS/PHEMA IPN samples. This study provides a strategy for using a biomacromolecules that can potentially improve cellular interactions.

\section{Acknowledgements}

The authors gratefully acknowledge Prof. Rahmah Noordin and Dr. Ong Ming Thong of the Institute for Research in Molecular Medicine (INFORMM) for their assistance with cell culture tests.

\section{References}

[1] Park J., Lakes R. S.: Biomaterials. Springer, New York (2007).

DOI: $10.1007 / 978-0-387-37880-0$

[2] Kirkpatrick C., Bittinger F., Wagner M., Köhler H., van Kooten T., Klein C., Otto M.: Current trends in biocompatibility testing. Proceedings of the Institution of Mechanical Engineers Part H: Journal of Engineering in Medicine, 212, 75-84 (1998). DOI: $10.1243 / 0954411981533845$

[3] Ř́íhová B.: Biocompatibility of biomaterials: Hemocompatibility, immunocompatiblity and biocompatibility of solid polymeric materials and soluble targetable polymeric carriers. Advanced Drug Delivery Reviews, 21, 157-176 (1996).

DOI: 10.1016/S0169-409X(96)00404-8

[4] Khorasani M. T., Mirzadeh H., Sammes P. G.: Laser induced surface modification of polydimethylsiloxane as a super-hydrophobic material. Radiation Physics and Chemistry, 47, 881-888 (1996).

DOI: 10.1016/0969-806X(95)00166-U

[5] Vladkova T.: Surface modification of silicone rubber with poly(ethylene glycol) hydrogel coatings. Journal of Applied Polymer Science, 92, 1486-1492 (2004). DOI: 10.1002/app.20001 
[6] Cifcová I., Lopour P., Vondráček P., Jelínek F.: Silicone rubber-hydrogel composites as polymeric biomaterials: I. Biogical properties of the silicone rubberp(HEMA) composite. Biomaterials, 11, 393-396 (1990). DOI: 10.1016/0142-9612(90)90093-6

[7] Griffith L. G.: Polymeric biomaterials. Acta Materialia, 48, 263-277 (2000).

DOI: 10.1016/S1359-6454(99)00299-2

[8] Lee C. H., Singla A., Lee Y.: Biomedical applications of collagen. International Journal of Pharmaceutics, 221, 1-22 (2001).

DOI: 10.1016/S0378-5173(01)00691-3

[9] Yue Z., Liu X., Molino P. J., Wallace G. G.: Bio-functionalisation of polydimethylsiloxane with hyaluronic acid and hyaluronic acid - Collagen conjugate for neural interfacing. Biomaterials, 32, 4714-4724 (2011). DOI: 10.1016/j.biomaterials.2011.03.032

[10] Bisson I., Kosinski M., Ruault S., Gupta B., Hilborn J., Wurm F., Frey P.: Acrylic acid grafting and collagen immobilization on poly(ethylene terephthalate) surfaces for adherence and growth of human bladder smooth muscle cells. Biomaterials, 23, 3149-3158 (2002).

DOI: $10.1016 / \mathrm{S} 0142-9612(02) 00061-3$

[11] Cheng Z., Teoh S-H.: Surface modification of ultra thin poly ( $\varepsilon$-caprolactone) films using acrylic acid and collagen. Biomaterials, 25, 1991-2001 (2004).

DOI: $10.1016 /$ j.biomaterials.2003.08.038

[12] Li Y-H., Huang Y-D.: The study of collagen immobilization on polyurethane by oxygen plasma treatment to enhance cell adhesion and growth. Surface and Coatings Technology, 201, 5124-5127 (2007).

DOI: $10.1016 /$ j.surfcoat.2006.07.128

[13] Ma L., Gao C., Mao Z., Zhou J., Shen J., Hu X., Han C.: Collagen/chitosan porous scaffolds with improved biostability for skin tissue engineering. Biomaterials, 24, 4833-4841 (2003).

DOI: $10.1016 / \mathrm{S} 0142-9612(03) 00374-0$

[14] Yan T., Sun R., Li C., Tan B., Mao X., Ao N.: Immobilization of type-I collagen and basic fibroblast growth factor (bFGF) onto poly (HEMA-co-MMA) hydrogel surface and its cytotoxicity study. Journal of Materials Science: Materials in Medicine, 21, 2425-2433 (2010). DOI: $10.1007 / \mathrm{s} 10856-010-4094-5$

[15] Hu S-G., Jou C-H., Yang M-C.: Biocompatibility and antibacterial activity of chitosan and collagen immobilized poly(3-hydroxybutyric acid-co-3-hydroxyvaleric acid). Carbohydrate Polymers, 58, 173-179 (2004).

DOI: $10.1016 /$ j.carbpol.2004.06.039

[16] Chirila T. V., Constable I. J., Crawford G. J., Vijayasekaran S., Thompson D. E., Chen Y-C., Fletcher W. A., Griffin B. J.: Poly(2-hydroxyethyl methacrylate) sponges as implant materials: In vivo and in vitro evaluation of cellular invasion. Biomaterials, 14, 2637 (1993). DOI: 10.1016/0142-9612(93)90072-A
[17] Hoffman A. S.: Hydrogels for biomedical applications. Advanced Drug Delivery Reviews, 54, 3-12 (2002). DOI: $10.1016 / \mathrm{S} 0169-409 X(01) 00239-3$

[18] Hsiue G-H., Guu J-A., Cheng C-C.: Poly(2-hydroxyethyl methacrylate) film as a drug delivery system for pilocarpine. Biomaterials, 22, 1763-1769 (2001). DOI: $10.1016 / \mathrm{S} 0142-9612(00) 00336-7$

[19] Rosiak J. M., Yoshii F.: Hydrogels and their medical applications. Nuclear Instruments and Methods in Physics Research Section B: Beam Interactions with Materials and Atoms, 151, 56-64 (1999). DOI: 10.1016/S0168-583X(99)00118-4

[20] Young C-D., Wu J-R., Tsou T-L.: Fabrication and characteristics of polyHEMA artificial skin with improved tensile properties. Journal of Membrane Science, 146, 83-93 (1998). DOI: $10.1016 / \mathrm{S} 0376-7388(98) 00097-0$

[21] Chetoni P., Di Colo G., Grandi M., Morelli M., Saettone M. F., Darougar S.: Silicone rubber/hydrogel composite ophthalmic inserts: Preparation and preliminary in vitro/in vivo evaluation. European Journal of Pharmaceutics and Biopharmaceutics, 46, 125-132 (1998). DOI: $10.1016 / \mathrm{S} 0939-6411(97) 00168-9$

[22] Hsiue G-H., Lee S-D., Wang C-C., Shiue M. H-I., Chang P. C-T.: ppHEMA-modified silicone rubber film towards improving rabbit corneal epithelial cell attachment and growth. Biomaterials, 14, 591-597 (1993). DOI: 10.1016/0142-9612(93)90177-4

[23] Khorasani M. T., Mirzadeh H., Sammes P. G.: Laser surface modification of polymers to improve biocompatibility: HEMA grafted PDMS, in vitro assay - III. Radiation Physics and Chemistry, 55, 685-689 (1999). DOI: 10.1016/S0969-806X(99)00212-1

[24] Lednický F., Janatová V., Lopour P., Vondráček P.: Silicone rubber-hydrogel composites as polymeric biomaterials III. An investigation of phase distribution by scanning electron microscopy. Biomaterials, 12, 848852 (1991). DOI: 10.1016/0142-9612(91)90073-J

[25] Lopour P., Vondráček P., Janatová V., Šulc J., Vacik J.: Silicone rubber-hydrogel composites as polymeric biomaterials: II. Hydrophilicity and permeability to watersoluble low-molecular-weight compounds. Biomaterials, 11, 397-402 (1990). DOI: 10.1016/0142-9612(90)90094-7

[26] Okada T., Ikada Y.: Modification of silicone surface by graft polymerization of acrylamide with corona discharge. Die Makromolekulare Chemie, 192, 17051713 (1991). DOI: 10.1002/macp.1991.021920804

[27] Rezaei S. M., Mohd Ishak Z. A.: The biocompatibility and hydrophilicity evaluation of collagen grafted poly (dimethylsiloxane) and poly (2-hydroxyethylmethacrylate) blends. Polymer Testing, 30, 69-75 (2011). DOI: $10.1016 /$ j.polymertesting.2010.07.013 
[28] Abbasi F., Mirzadeh H., Katbab A-A.: Modification of polysiloxane polymers for biomedical applications: A review. Polymer International, 50, 1279-1287 (2001). DOI: $10.1002 /$ pi. 783

[29] Fichet O., Vidal F., Laskar J., Teyssie D.: Polydimethylsiloxane-cellulose acetate butyrate interpenetrating polymer networks synthesis and kinetic study. Part I. Polymer, 46, 37-47 (2005).

DOI: $10.1016 /$ j.polymer.2004.10.053

[30] Park S., Nam S. H., Koh W-G.: Preparation of collagen-immobilized poly(ethylene glycol)/poly(2-hydroxyethyl methacrylate) interpenetrating network hydrogels for potential application of artificial cornea. Journal of Applied Polymer Science, 123, 637-645 (2011). DOI: 10.1002/app.34532
[31] Wang J., Li X.: Enhancing protein resistance of hydrogels based on poly(2-hydroxyethyl methacrylate) and poly(2-methacryloyloxyethyl phosphorylcholine) with interpenetrating network structure. Journal of Applied Polymer Science, 121, 3347-3352 (2011).

DOI: 10.1002/app.33960

[32] Sperling L. H.: Interpenetrating polymer networks: An overview. Advances in Chemistry, 239, 3-38 (1994). DOI: 10.1021/ba-1994-0239.ch001

[33] Sperling L. H., Mishra V.: Metastable phase diagrams for simultaneous interpenetrating networks. Macromolecular Symposia, 118, 363-370 (1997).

DOI: $10.1002 /$ masy. 19971180147 